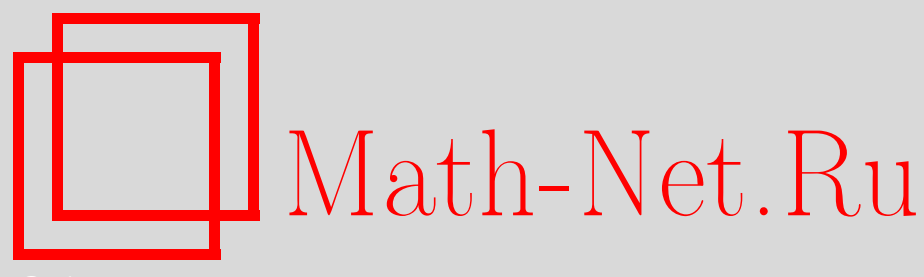

М. В. Павлов, Д. В. Прохоров, А. Ю. Васильев, А. М. Захаров, Эволюция Лёвнера и конечномерные редукции интегрируемых систем, ТМФ, 2014, том 181, номep 1, 155-172

DOI: https://doi.org/10.4213/tmf8724

Использование Общероссийского математического портала Math-Net.Ru подразумевает, что вы прочитали и согласны с пользовательским соглашением http: //www . mathnet.ru/rus/agreement

Параметры загрузки:

IP : 3.80 .181 .102

26 апреля 2023 г., 11:03:50

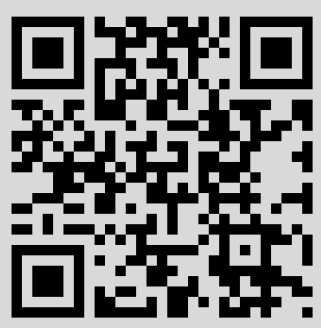




\title{
ФИЗИКА
}

Том 181, № 1

октябрь, 2014

(C) 2014 г.

М. В. Павлов ${ }^{* \dagger}$, Д. В. Прохоров ${ }^{\ddagger}$, А. Ю. Васильев ${ }^{\S}$ А. М. Захаров ${ }^{\ddagger}$

\section{ЭВОЛЮЦИЯ ЛЁВНЕРА И КОНЕЧНОМЕРНЫЕ РЕДУКЦИИ ИНТЕГРИРУЕМЫХ СИСТЕМ}

\begin{abstract}
Уравнение Лёвнера известно как одномерная редукция цепочки Бенни, а также как бездисперсионная иерархия Кадомцева-Петвиашвили. Предложена обратная процедура и показано, что расщепление времени в уравнении Лёвнера или в уравнении Лёвнера-Куфарева приводит к некоторым известным интегрируемым системам.
\end{abstract}

Ключевые слова: уравнение Лёвнера, интегрируемая система, уравнение Власова, моменты Бенни, бесстолкновительное кинетическое уравнение, гамильтонова структура, гидродинамическая цепочка, гидродинамическая редукция.

DOI: $10.4213 / \operatorname{tmf} 8724$

Посвящается Л.Д. Фаддееву по случаю его восъмидесятилетия

\section{1. ВВЕДЕНИЕ}

Одной из центральных проблем теории интегрируемых систем является их конечномерная редукция. Начнем в качестве примера с иерархии бездисперсионного уравнения Кадомцева-Петвиашвили (иерархии бКП). Пусть $\lambda(z, \mathbf{t})$ - мероморфная функция переменной $z$, зависящая также от бесконечного семейства обобщенных времен $\mathbf{t}=\left(t_{0}, t_{1}, \ldots, t_{n} \ldots\right), t_{0}=x$, которая в окрестности бесконечности разлагается в ряд

$$
\lambda(z, \mathbf{t})=z+\sum_{n=0}^{\infty} \frac{A^{n}(\mathbf{t})}{z^{n+1}}
$$

${ }^{*}$ Физический институт им. П. Н. Лебедева РАН, Москва, Россия. E-mail: maksmath@gmail.com

${ }^{\dagger}$ Московский инженерно-физический институт (государственный университет), Москва, Россия

${ }^{\ddagger}$ Саратовский государственный университет, Саратов, Россия. E-mail: prokhorov@sgu.ru, zakharovam66@gmail.com

${ }^{\S}$ Department of Mathematics, University of Bergen, Bergen, Norway.

E-mail: Alexander.Vasiliev@math.uib.no 
(здесь верхний индекс в выражении $A^{n}$ означает не степень, а индекс). Структуру Пуассона $\{\cdot, \cdot\}$ введем следующим образом:

$$
\{F, G\}=\frac{\partial F}{\partial z} \frac{\partial G}{\partial x}-\frac{\partial F}{\partial x} \frac{\partial G}{\partial z} .
$$

Тогда иерархия бКП (см. работу [1]) представляет собой бесконечное множество коммутирующих потоков

$$
\frac{\partial \lambda}{\partial t_{n}}=\left\{\mathcal{L}_{n+1}, \lambda\right\}, \quad n=0,1,2, \ldots
$$

где

$$
\mathcal{L}_{n}=\frac{1}{n}\left(\lambda^{n}\right)_{\geqslant 0}, \quad n=1,2, \ldots,
$$

означает полиномиальную часть выражения $\lambda^{n}$. В частности,

$$
(\lambda)_{\geqslant 0}=z, \quad\left(\lambda^{2}\right)_{\geqslant 0}=z^{2}+2 A^{0}, \quad\left(\lambda^{3}\right)_{\geqslant 0}=z^{3}+3 z A^{0}+3 A^{1} .
$$

При $n=2$ и $s=t_{1}$ второе уравнение иерархии эквивалентно уравнению

$$
A_{s}^{n}+A_{x}^{n+1}+n A^{n-1} A_{x}^{0}=0
$$

которое известно как уравнение моментов Бенни. Бенни исследовал [2] длинные нелинейные волны, распространяющиеся на свободной поверхности, и показал, что уравнения движения обладают бесконечным числом законов сохранения.

Условие совместности системы (1) имеет вид

$$
\frac{\partial \mathcal{L}_{m}}{\partial t_{n}}-\frac{\partial \mathcal{L}_{n}}{\partial t_{m}}+\left\{\mathcal{L}_{m}, \mathcal{L}_{n}\right\}=0
$$

и означает, что потоки (1) коммутируют. В частности, при $m=3, n=2, y=t_{2}$ и $u:=A^{0}$ получим уравнение

$$
u_{s s}+\left(u_{y}+u u_{x}\right)_{x}=0
$$

известное как уравнение бКП или уравнение Заболотской-Хохлова [3].

Конечномерная редукция предполагает, что функция $\lambda$ зависит от обобщенных времен $\mathbf{t}$ через конечное число функций $u_{k}=u_{k}(\mathbf{t})$, т. е.

$$
\lambda(z, \mathbf{t})=\lambda(z, \mathbf{u}(\mathbf{t}))=\lambda\left(z, u_{1}(\mathbf{t}), \ldots, u_{N}(\mathbf{t})\right) .
$$

Хорошо известная полиномиальная редукция была предложена Захаровым [4].

Кодама и Гиббонс [1] поняли, что уравнение бКП обладает бесконечным числом многокомпонентных двумерных редукций, и предложили несколько примеров, причем для этих частных случаев была найдена функция $\lambda$. В работе [1] была рассмотрена вектор-функция $\mathbf{u}$, удовлетворяющая системе гидродинамического типа

$$
\frac{\partial \mathbf{u}}{\partial t_{n}}=\xi_{n}(\mathbf{u}) \frac{\partial \mathbf{u}}{\partial x}, \quad n=2,3, \ldots
$$


Гиббонс и Царев [5] первыми обратили внимание на то, что хордовое уравнение Лёвнера играет важную роль в классификации редукций уравнений Бенни. Если ввести обозначения

$$
u=u_{0}=A^{0}, \ldots, u_{N}=A^{N}, \quad A^{n}=A^{n}(\mathbf{u}), \quad n>N,
$$

то мы получим $N(N-1) / 2$ условий совместности для $A^{N+1}$, при этом функция $z=z(\lambda, \mathbf{t})$, обратная к $\lambda$, удовлетворяет уравнениям

$$
\partial_{k} z=-\frac{\partial_{k} u}{z-\mu_{k}}
$$

где $\partial_{k}=\partial / \partial r^{k}, \mu_{k}$ - нули функции $\lambda_{z}$, а величины $r^{k}=\lambda\left(\mu_{k}, \mathbf{t}\right)$ являются инвариантами Римана. Приведенное выше уравнение с формальной точки зрения аналогично хордовому уравнению Лёвнера, которое обсуждается далее. Условия совместности для уравнений (5) имеют вид

$$
\partial_{i} \mu_{k}=\frac{\partial_{k} u}{\mu_{i}-\mu_{k}}, \quad \partial_{i} \partial_{k} u=2 \frac{\partial_{i} u \partial_{k} u}{\left(\mu_{i}-\mu_{k}\right)^{2}}
$$

и представляют собой систему Гиббонса-Царева.

В работе [6] было предложено обобщение, в котором функция $\lambda$ и обратная к ней ищутся как решения системы уравнений

$$
\frac{\partial z}{\partial u_{i}}=\sum_{k=1}^{N} \frac{\eta_{i k}}{z-\mu_{k}},
$$

удовлетворяющей некоторым условиям совместности. С формальной точки зрения приведенное выше уравнение снова имеет вид хордового уравнения Лёвнера со многими разрезами.

Позже Такебе, Тео и Забродин [7] показали, что хордовое и радиальное уравнения Лёвнера являются условиями совместности редукций к одной переменной для иерархий бКП и цепочки Тоды соответственно. В хордовом случае функция $\lambda$, удовлетворяющая равенству (1), зависит от $\mathbf{t}$ посредством одной функции $s(\mathbf{t})$, причем

$$
\frac{\partial \lambda}{\partial s}=-\frac{\kappa}{z-\xi} \frac{\partial \lambda}{\partial z}
$$

где $\kappa$ является производной по $s$ коэффициента при $1 / z$ в разложении Лорана функции $\lambda$. Кроме того, имеет место условие совместности гидродинамического типа

$$
\frac{\partial s}{\partial t_{n}}=\chi_{n} \frac{\partial s}{\partial x}
$$

и функции $\chi_{n}(s)$ строятся каноническим образом по функциям Лакса. Мы снова получаем хордовое уравнение Лёвнера в частных производных. Эти подходы в определенной степени нуждаются в аналитическом объяснении, которое можно вывести из теории Лёвнера.

С другой стороны, еще один эволюционный процесс, описанный лапласовским ростом [8], обладает бесконечным числом законов сохранения, выраженных гармоническими моментами [9]. Моменты лапласовского роста удовлетворяют бездисперсионной иерархии Тоды [10]. В отличие от лапласовского роста, эволюция Лёвнера 
относится к другой группе моделей, когда эволюция обусловлена бесконечным числом управляющих параметров, а именно к группе контролируемых динамических систем, в которых бесконечное число степеней свободы связано с бесконечным числом управляющих членов. Удивительно, что для этой группы появляется та же самая структурная основа - алгебра Вирасоро [11].

Идея настоящей статьи состоит в пересмотре выводов Гиббонса и Царева с целью показать, что хордовая эволюция Лёвнера также обладает бесконечным числом законов сохранения (моментов). Мы покажем, что уравнение Лёвнера в частных производных представляет собой в точности уравнение Власова, если использовать соответствующую замену переменных, а обыкновенное дифференциальное уравнение Лёвнера имеет следствием закон сохранения гидродинамического типа. Мы начнем с эволюции Лёвнера и, расщепив время, получим интегрируемые цепочки. Этот подход демонстрирует универсальность уравнения Лёвнера как объединяющего механизма для нескольких интегрируемых цепочек, что было отмечено в работе [12].

\section{2. УРАВНЕНИЯ ВЛАСОВА И ЛЁВНЕРА, ЗАКОНЫ СОХРАНЕНИЯ}

Рассмотрим цепь Лёвнера сжимающихся областей $\mathbb{H}_{t}=\mathbb{H} \backslash \gamma_{t}$ в верхней полуплоскости $\mathbb{H}=\{z: \operatorname{Im} z>0\}$ (см. рис. 1). Пусть функция $f: \mathbb{H} \rightarrow \mathbb{H}_{t}$ нормирована в окрестности бесконечности следующим образом:

$$
f(z, t)=z+\frac{A^{0}}{z}+O\left(\frac{1}{z^{2}}\right)
$$

где $-A^{0}(t)$ есть емкость кривой $\gamma_{t}$ относительно полуплоскости. Пусть $\gamma_{t}-$ кривая Жордана, целиком лежащая в полуплоскости $\mathbb{H}$, за исключением конечной точки на действительной оси $\mathbb{R}$, кривая $\gamma_{t}$ параметризуется временем $t$. Тогда $f$ удовлетворяет уравнению Лёвнера в частных производных

$$
\left(z-\xi_{t}\right) \frac{\partial f(z, t)}{\partial t}-\frac{d A^{0}}{d t} \frac{\partial f(z, t)}{\partial z}=0
$$

с вещественнозначной непрерывной управляющей функцией $\xi_{t}$ и начальным условием $f(z, 0)=f_{0}(z)$. Для каждого $t \geqslant 0$ функция $f(z, t)$ имеет непрерывное продолжение на замыкание полуплоскости $\mathbb{H}$, причем продолженная функция, которую мы также обозначим через $f(z, t)$, удовлетворяет уравнению (8), по крайней мере, почти всюду. Управляющая функция $\xi_{t}$ порождает растущий разрез $\gamma_{t}$.

Воспользуемся двухпараметрическим семейством конформных отображений

$$
g(w, t, \tau):=f^{-1}(w(z, t), \tau)=f^{-1}(f(z, t), \tau), \quad 0 \leqslant \tau \leqslant t<\infty .
$$

Положим также $g(w, t, 0)=: g(w, t)$. Функция $g$ отображает полуплоскость $\mathbb{H}$ в некоторое ее подмножество и удовлетворяет обыкновенному дифференциальному уравнению Лёвнера на полуплоскости:

$$
\frac{\partial g(w, t, \tau)}{\partial t}=-\frac{1}{g(w, t, \tau)-\xi_{t}} \frac{d A^{0}}{d t}, \quad 0 \leqslant \tau \leqslant t<\infty, \quad g(w, \tau, \tau)=w .
$$

Более того,

$$
\lim _{t \rightarrow \infty} g(w, t, \tau)=\lim _{t \rightarrow \infty} f^{-1}(f(z, t), \tau)=f(z, \tau) .
$$




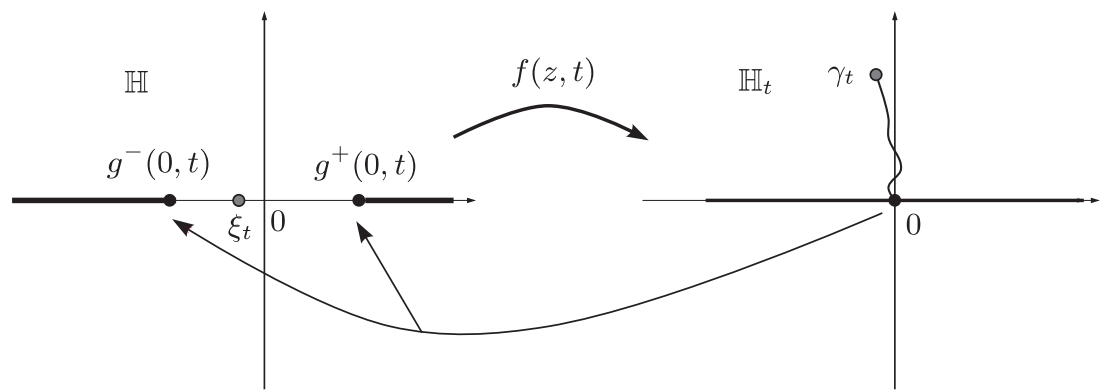

Рис. 1. Цепь Лёвнера сжимающихся областей.

Введем расщепление времени как вещественнозначную функцию $t=t(x, s)$, которая является решением квазилинейного дифференциального уравнения

$$
\xi_{t} \frac{\partial t}{\partial x}+\frac{\partial t}{\partial s}=0
$$

с асимптотическим поведением

$$
\lim _{x \rightarrow \infty} t(x, s)=\lim _{x \rightarrow-\infty} t(x, s)<\infty .
$$

Предположим, что $\xi_{t}$ является функцией, допускающей конус решений для уравнения (10) с нужным асимптотическим поведением.

Рассмотрим теперь суперпозицию $f(z, t(x, s))$ и умножим обе части уравнения (8) на $\partial t / \partial x$. Чтобы избежать дополнительных обозначений, мы продолжим писать $f$ вместо $f(z, t(x, s))=f(z, x, s)$. Тогда

$$
z \frac{\partial f}{\partial x}-\xi_{t} \frac{\partial f}{\partial t} \frac{\partial t}{\partial x}-\frac{\partial A^{0}}{\partial x} \frac{\partial f}{\partial z}=0 .
$$

Используя (10), получим уравнение

$$
z \frac{\partial f}{\partial x}+\frac{\partial f}{\partial s}-\frac{\partial A^{0}}{\partial x} \frac{\partial f}{\partial z}=0
$$

которое представляет собой уравнение Власова [5], [13]. Это уравнение описывает эволюцию функции распределения плазмы, состоящей из заряженных частиц с дальним взаимодействием. В гидродинамическом описании плазмы рассматривается распределение не скоростей, а моментов $A^{n}(t(x, s)) \equiv A^{n}(x, s)$ плазмы.

Выберем среди решений уравнения Власова (11) такие, которые дают конечные интегралы для моментов $A^{n}$. А именно, для данного решения $f(z, x, s)$ с нормировкой (7) выберем решение $\phi(z, x, s)=\varphi(f(z, x, s))$, где $\varphi$ - подходящая быстро убывающая при $z \rightarrow \pm \infty$ функция (см., например, работу [14]). В качестве такой функции можно выбрать, скажем, $\varphi(f)=e^{-f^{2}}$. Тогда моменты $A^{n}(x, s)$ определим следующим образом:

$$
A^{n}(x, s)=\int_{-\infty}^{\infty} w^{n} \phi(w, x, s) d w, \quad n=1,2, \ldots
$$


С помощью непосредственных вычислений получим

$$
A_{s}^{n}=\int_{-\infty}^{\infty} w^{n} \frac{\partial \phi}{\partial s} d w, \quad A_{x}^{n+1}=\int_{-\infty}^{\infty} w^{n+1} \frac{\partial \phi}{\partial x} d w .
$$

Интегрируя по частям, имеем

$$
A^{n-1}=-\int_{-\infty}^{\infty} \frac{w^{n}}{n} \frac{\partial \phi}{\partial w} d w
$$

Теперь можно воспользоваться уравнением Власова (11) и получить уравнения для моментов

$$
A_{s}^{n}+A_{x}^{n+1}+n A^{n-1} A_{x}^{0}=0
$$

которые представляют собой бесконечную автономную систему, известную как уравнения моментов Бенни [2]. Данная система появляется в длинноволновой гидродинамике идеальной несжимаемой жидкости конечной глубины в гравитационном поле.

Следуя работам [5], [15], введем функцию $\lambda(z, x, s)$ с помощью главного значения Коши сингулярного интеграла:

$$
\lambda(z, x, s)=z+\int_{-\infty}^{\infty} \frac{\phi(w, x, s)}{z-w} d w=z+\sum_{n=0}^{\infty} \frac{A^{n}}{z^{n+1}}, \quad z \in \mathbb{H}, \quad z \rightarrow \infty
$$

где $z=g(w, t(x, s))$, а коэффициент $A^{0}$ тот же, что и для $f$. Тогда

$$
\begin{aligned}
& \lambda_{s}=\frac{\partial \lambda}{\partial s}=z_{s}+\sum_{n=0}^{\infty}\left(\frac{A_{s}^{n}}{z^{n+1}}-\frac{(n+1) A^{n} z_{s}}{z^{n+2}}\right), \\
& \lambda_{x}=\frac{\partial \lambda}{\partial x}=z_{x}+\sum_{n=0}^{\infty}\left(\frac{A_{x}^{n}}{z^{n+1}}-\frac{(n+1) A^{n} z_{x}}{z^{n+2}}\right)
\end{aligned}
$$

и

$$
\lambda_{s}+z \lambda_{x}=z_{s}+z z_{x}+A_{x}^{0}+\sum_{n=0}^{\infty} \frac{A_{s}^{n}+A_{x}^{n+1}-n A^{n-1} z_{s}-(n+1) A^{n} z_{x}}{z^{n+1}} .
$$

Используя уравнение для моментов, приходим к соотношению

$$
\begin{aligned}
\lambda_{s}+z \lambda_{x} & =z_{s}+z z_{x}+A_{x}^{0}+\sum_{n=0}^{\infty} \frac{-n A^{n-1} A_{x}^{0}}{z^{n+1}}-\sum_{n=1}^{\infty} \frac{n A^{n-1}\left(z_{s}+z z_{x}\right)}{z^{n+1}}= \\
& =A_{x}^{0} \lambda_{z}+\left(z_{s}+z z_{x}\right)\left(1-\sum_{n=1}^{\infty} \frac{n A^{n-1}}{z^{n+1}}\right)=\lambda_{z}\left(A_{x}^{0}+z_{s}+z z_{x}\right) .
\end{aligned}
$$

Уравнение Лёвнера (9) влечет $A_{x}^{0}=z_{x}\left(\xi_{t}-z\right)$, а определение функции $t(x, s)$ приводит к соотношению

$$
A_{x}^{0}+z_{s}+z z_{x}=0
$$

следовательно, на траекториях уравнения Лёвнера (9) справедливо равенство

$$
\lambda_{s}+z \lambda_{x}=0
$$


В работе [16] уравнение (13) было названо уравнением Гиббонса в соответствии с оригинальной работой Гиббонса [17].

Рассмотрим отображение $z(\lambda, x, s)$, обратное отображению $\lambda(z, x, s)$ по отношению к переменным $\lambda \leftrightarrow z$,

$$
\begin{aligned}
& \lambda(z, x, s)=z+\sum_{n=0}^{\infty} \frac{A^{n}}{z^{n+1}}, \quad z \in \mathbb{H}, \quad z \rightarrow \infty \\
& z(\lambda, x, s)=\lambda-\sum_{n=0}^{\infty} \frac{H^{n}}{\lambda^{n+1}} .
\end{aligned}
$$

Тогда

$$
\sum_{n=0}^{\infty} \frac{A^{n}}{z^{n+1}}=\sum_{n=0}^{\infty} \frac{H^{n}}{\lambda^{n+1}}, \quad \frac{\lambda}{z}\left(A^{0}+\frac{A^{1}}{z}+\cdots\right)=H^{0}+\frac{H^{1}}{\lambda}+\cdots .
$$

Итак, $H^{0}=A^{0}$. Далее,

$$
\lambda\left(\frac{\lambda}{z}-1\right) A^{0}+\frac{\lambda^{2}}{z^{2}}\left(A^{1}+\frac{A^{2}}{z}+\cdots\right)=H^{1}+\frac{H^{2}}{\lambda}+\cdots,
$$

и мы приходим к выводу, что $H^{1}=A^{1}$. Аналогично,

$$
\lambda^{2}\left(\frac{\lambda}{z}-1\right) A^{0}+\lambda\left(\frac{\lambda^{2}}{z^{2}}-1\right) A^{1}+\frac{\lambda^{3}}{z^{3}}\left(A^{2}+\frac{A^{3}}{z}+\cdots\right)=H^{2}+\frac{H^{3}}{\lambda}+\cdots
$$

и $H^{2}=A^{2}+\left(A^{0}\right)^{2}$. Окончательно имеем

$$
\sum_{k=0}^{n} \lambda^{n-k}\left(\frac{\lambda^{k+1}}{z^{k+1}} A^{k}-H^{k}\right)+\frac{\lambda^{n+1}}{z^{n+2}}\left(A^{n+1}+\frac{A^{n+2}}{z}+\cdots\right)=\frac{1}{\lambda}\left(H^{n+1}+\frac{H^{n+2}}{\lambda}+\cdots\right),
$$

где коэффициент $H^{n}$ рассчитывается по формуле $H^{n}=A^{n}+P\left(A^{0}, \ldots, A^{n-1}\right)$, в которой $P\left(A^{0}, \ldots, A^{n-1}\right)$ - полином по $A^{0}, \ldots, A^{n-1}, n \geqslant 2$. Первые коэффициенты имеют вид

$$
\begin{gathered}
H^{0}=A^{0}, \quad H^{1}=A^{1}, \quad H^{2}=A^{2}+\left(A^{0}\right)^{2}, \quad H^{3}=A^{3}+3 A^{0} A^{1}, \\
H^{4}=A^{4}+4 A^{0} A^{2}+2\left(A^{1}\right)^{2}+2\left(A^{0}\right)^{3} .
\end{gathered}
$$

Аналогичные коэффициенты были вычислены в работах [15], [18].

Таким образом, уравнение Лёвнера (9) становится законом сохранения в следующим смысле. Согласно соотношению (13) имеем

$$
\frac{d}{d s} \int_{-\infty}^{\infty} z(\lambda, x, s) d x=-\int_{-\infty}^{\infty}\left(A_{x}^{0}+z z_{x}\right) d x
$$

где интеграл по $x \in \mathbb{R}$ понимается в смысле главного значения Коши. Требования к асимптотическому поведению функции $t(x, s)$ при $x \rightarrow \pm \infty$ подразумевают, что

$$
\int_{-\infty}^{\infty}\left(A_{x}^{0}+z z_{x}\right) d x=0,
$$

6 Теоретическая и математическая физика, т. 181, № 1, 2014 г. 
поэтому

$$
\frac{d}{d s} \int_{-\infty}^{\infty} z(\lambda, x, s) d x=0
$$

что соответствует закону сохранения моментов. Итак, величинами, сохраняющимися в процессе эволюции, являются моменты

$$
I^{n}=\int_{-\infty}^{\infty} H^{n}(x, s) d x, \quad n=0,1,2, \ldots .
$$

Аналогичные интегралы движения изучались в оригинальной работе Бенни [2], а также в работах [4], [15], [19].

Структура Пуассона позволяет переформулировать уравнение моментов Бенни (12) в виде эволюционного уравнения с гамильтоновой функцией. Пуассонова структура Купершмидта-Манина [15], [20] для моментов $A^{n} \frac{\partial}{\partial x}$ задается с помощью введения $L^{2}(\mathbb{R})$-кососимметрических операторов, действующих по формуле

$$
\left\{A^{m}, A^{n}\right\}(\cdot)=-m A^{n+m-1} \frac{\partial}{\partial x}(\cdot)-n \frac{\partial}{\partial x}\left(A^{n+m-1}(\cdot)\right) .
$$

Тогда для любых $F(A)$ и $G(A)$ можно представить скобку Пуассона в виде

$$
\{F, G\}(A)=\sum_{m, n=0}^{\infty} \int_{-\infty}^{\infty} \frac{\delta F}{\delta A^{m}}\left\{A^{m}, A^{n}\right\} \frac{\delta G}{\delta A^{n}} d x .
$$

Записав формулу

$$
\bar{H}^{k}=\frac{1}{k} \int_{-\infty}^{\infty} H^{k} d x=\frac{1}{k} I^{k}
$$

получим иерархию коммутирующих потоков в виде эволюционных уравнений с гамильтонианами $\bar{H}^{k}$ такими, что $\left\{\bar{H}^{k}, \bar{H}^{j}\right\}=0$ :

$$
\frac{\partial A^{m}}{\partial t_{k}}=\sum_{n=0}^{\infty}\left\{A^{m}, A^{n}\right\} \frac{\delta \bar{H}^{k}}{\delta A^{n}} .
$$

Таким образом, уравнение (12) становится вторым уравнением из этой иерархии.

\section{3. КОНЕЧНОМЕРНОЕ ВРЕМЯ}

Уравнение Лёвнера (8) в частных производных можно обобщить до уравнения:

$$
\frac{\partial f(z, t)}{\partial t}=\sum_{k=1}^{m} \frac{\mu_{k}(t)}{z-\xi_{k}(t)} \frac{d A^{0}}{d t} \frac{\partial f(z, t)}{\partial z}, \quad f(z, 0)=f_{0}(z)
$$

с кусочно-непрерывными коэффициентами $\mu_{k}(t) \geqslant 0, k=1, \ldots, m$, такими, что $\sum_{k=1}^{m} \mu_{k}(t)=1$, и вещественнозначными непрерывными управляющими функциями $\xi_{1}(t), \ldots, \xi_{m}(t)$. Решение $f(z, t)$ уравнения (16) отображает полуплоскость $\mathbb{H}$ в $\mathbb{H} \backslash \cup_{k=1}^{m} \gamma_{k}(t)$, где $\gamma_{k}(t)$ - растущие кривые Жордана (разрезы), лежащие в $\mathbb{H}$, за исключением их конечных точек на $\mathbb{R}$. Управляющие функции $\xi_{k}(t)$ порождают разрезы $\gamma_{k}(t)$, а коэффициенты $\mu_{k}(t)$ управляют динамикой разрезов $\gamma_{k}(t)$ относительно друг друга. 
Вместо уравнения (16) можно рассмотреть обобщенное уравнение Лёвнера в частных производных с обобщенным вектором времени $\mathbf{t}=\left(t_{1}, \ldots, t_{m}\right)$ :

$$
\left(z-\xi_{k}(\mathbf{t})\right) \frac{\partial f(z, \mathbf{t})}{\partial t_{k}}=\frac{\partial A^{0}}{\partial t_{k}} \frac{\partial f(z, \mathbf{t})}{\partial z}, \quad k=1, \ldots, m, \quad f(z, \mathbf{0})=f_{\mathbf{0}}(z),
$$

где $\mathbf{0}$ означает нулевой вектор. В этой модели $A^{0}(\mathbf{t})=A^{0}\left(t_{1}, \ldots, t_{m}\right)$ не является произвольной функцией от $\mathbf{t} . \mathrm{B}$ каждой точке $\mathbf{t}=\left(t_{1}, \ldots, t_{m}\right)$ имеем

$$
\frac{\partial A^{0}}{\partial t_{1}}=\frac{\partial A^{0}}{\partial t_{2}}=\cdots=\frac{\partial A^{0}}{\partial t_{m}}
$$

Для каждой точки $\mathbf{t}=\left(t_{1}, \ldots, t_{m}\right)$, решение $f(z, \mathbf{t})$ системы (17) отображает полуплоскость $\mathbb{H}$ в $\mathbb{H} \backslash \cup_{k=1}^{m} \gamma_{k}\left(t_{k}\right)$, где $\gamma_{k}\left(t_{k}\right)$ является конечной точкой разреза $\gamma_{k}$, порожденного управляющей функцией $\xi_{k}(\mathbf{t})$.

Аналогично случаю скалярного $t$ положим

$$
g(w, \tau, \mathbf{t}):=f^{-1}(w(z, \tau), \mathbf{t})=f^{-1}(f(z, \tau), \mathbf{t}), \quad \tau=\left(\tau_{1}, \ldots, \tau_{m}\right),
$$

где $0 \leqslant \tau_{k} \leqslant t_{k}<\infty$ для любого $k=1, \ldots, m$. Мы также введем $g(w, \mathbf{0}, \mathbf{t})=: g(w, \mathbf{t})$. Функция $g$ отображает полуплоскость $\mathbb{H}$ в ее подмножество и удовлетворяет системе обыкновенных дифференциальных уравнений Лёвнера на полуплоскости

$$
\begin{aligned}
\frac{\partial g(w, \tau, \mathbf{t})}{\partial t_{k}} & =-\frac{1}{g(w, \tau, \mathbf{t})-\xi_{k}(\mathbf{t})} \frac{\partial A^{0}}{\partial t_{k}}, \quad k=1, \ldots, m, \\
g(w, \tau, \tau) & =w
\end{aligned}
$$

здесь $\tau_{j} \leqslant t_{j}<\infty$ для всех $j=1, \ldots, m$. Более того,

$$
\lim _{t_{k} \rightarrow \infty} g\left(w, \tau,\left(\tau_{1}, \ldots, \tau_{k-1}, t_{k}, \tau_{k+1}, \ldots, \tau_{m}\right)\right)=f(z, \tau) .
$$

Вектор-функцию $\mathbf{t}=\mathbf{t}(x, s)$ можно снова ввести как решение системы квазилинейных дифференциальных уравнений

$$
\xi_{k}(\mathbf{t}) \frac{\partial t_{k}}{\partial x}+\frac{\partial t_{k}}{\partial s}=0, \quad k=1, \ldots, m,
$$

с асимптотическим поведением

$$
\lim _{x \rightarrow \infty} t_{k}(x, s)=\lim _{x \rightarrow-\infty} t_{k}(x, s)<\infty, \quad k=1, \ldots, m .
$$

Предположим, что функции $\xi_{k}\left(t_{k}\right)$ допускают конусы решений уравнения $(19)$ с необходимым асимптотическим поведением.

Уравнение (17) подразумевает, что функция $f(z, \mathbf{t}(x, s))=: f(z, x, s)$ удовлетворяет уравнению

$$
z \frac{\partial f}{\partial t_{k}} \frac{\partial t_{k}}{\partial x}-\xi_{k}(\mathbf{t}) \frac{\partial f}{\partial t_{k}} \frac{\partial t_{k}}{\partial x}-\frac{\partial A^{0}}{\partial t_{k}} \frac{\partial t_{k}}{\partial x} \frac{\partial f}{\partial z}=0
$$

которое вместе с (19) дает

$$
z \frac{\partial f}{\partial t_{k}} \frac{\partial t_{k}}{\partial x}+\frac{\partial f}{\partial t_{k}} \frac{\partial t_{k}}{\partial s}-\frac{\partial A^{0}}{\partial t_{k}} \frac{\partial t_{k}}{\partial x} \frac{\partial f}{\partial z}=0, \quad k=1, \ldots, m .
$$


Суммируя последние уравнения по $k=1, \ldots, m$, получим уравнение Власова для функции $f(z, \mathbf{t}(x, s))$ :

$$
z \frac{\partial f(z, \mathbf{t}(x, s))}{\partial x}+\frac{\partial f(z, \mathbf{t}(x, s))}{\partial s}-\frac{\partial A^{0}}{\partial x} \frac{\partial f(z, \mathbf{t}(x, s))}{\partial z}=0 .
$$

Аналогично скалярному случаю появляются моменты $A^{n}(\mathbf{t}(x, s))=: A^{n}(x, s)$, удовлетворяющие уравнению (12), функции $\lambda(z, x, s)$ и $z(\lambda, x, s)$, а также иерархия коммутирующих потоков с гамильтонианами $\bar{H}^{k}$.

Уравнения (17) можно свести к уравнению (16). В самом деле, предположим, что $\mu_{1}(t)>0$, и построим следующую редукцию:

$$
\frac{d t_{k}}{d t_{1}}=\frac{\mu_{k}}{\mu_{1}}, \quad t_{k}(0)=0, \quad k=2, \ldots, m .
$$

Тогда, после умножения на $\partial t_{k} / \partial t_{1}$ и суммирования, уравнения (17) для $t_{1}=t$ и $f(z, \mathbf{t}(t))=: f(z, t)$ переходят в уравнение

$$
\frac{\partial f(z, \mathbf{t}(t))}{\partial t}=\frac{\partial f}{\partial t_{1}}+\frac{\partial f}{\partial t_{2}} \frac{\partial t_{2}}{\partial t}+\cdots+\frac{\partial f}{\partial t_{m}} \frac{\partial t_{m}}{\partial t}=\frac{1}{\mu_{1}}\left[\frac{\mu_{1}}{z-\xi_{1}} \frac{\partial A^{0}}{\partial t_{1}}+\cdots+\frac{\mu_{m}}{z-\xi_{m}} \frac{\partial A^{0}}{\partial t_{m}}\right] \frac{\partial f}{\partial z},
$$

которое эквивалентно уравнению (16) с $\tilde{A}^{0}=A^{0} / \mu_{1}$.

\section{4. БЕСКОНЕЧНОМЕРНОЕ ВРЕМЯ}

Предельный случай уравнения (16) при $m \rightarrow \infty$ приводит к уравнению типа Лёвнера-Куфарева

$$
\frac{\partial f}{\partial t}=\int_{\mathbb{R}} \frac{d A^{0}}{d t} \frac{\partial f}{\partial z} \frac{d \nu_{t}(\xi)}{z-\xi(t)}
$$

где для любого $t \geqslant 0$ величина $d \nu_{t}(\xi)$ является вероятностной мерой с компактным носителем $I_{t} \subset \mathbb{R}$. Решение $f(z, t)$ уравнения $(20)$ отображает полуплоскость $\mathbb{H}$ в $\mathbb{H} \backslash K_{t}$, где, вообще говоря, отброшенное множество $K_{t} \cap \mathbb{H}$ не сводится к счетному набору разрезов. Множество $K_{t}$ порождается мерой $d \nu_{t}(\xi)$. Однако область $\mathbb{H} \backslash K_{t}$ является ядром Каратеодори для последовательности областей $\mathbb{H} \backslash \cup_{k=1}^{m} \gamma_{k}(t)$ при $m \rightarrow \infty$, причем разрезы $\gamma_{k}$ плотны в $K_{t}$. В такой интерпретации мера $d \nu_{t}(\xi)$ представляется как предел мер точечных масс с плотным множеством точечных масс на носителе вероятностной меры $d \nu_{t}$. В этом случае невозможно напрямую обобщить систему (17), переходя к бесконечному набору уравнений, соответствующих счетному набору координат $\left(t_{1}, t_{2}, \ldots\right)$.

Построим модель с последовательной динамикой каждого из разрезов $\gamma_{1}, \gamma_{2}, \ldots$ Для $k=1,2, \ldots$ введем обозначение

$$
P_{k}\left(z, t_{k}\right)=\left\{\begin{array}{lll}
\frac{\partial A^{0}}{\partial t_{k}} \frac{\partial f\left(z, t_{k}\right)}{\partial z} & \text { при } & T_{k-1}<t_{k}<T_{k} \\
0 & \text { при } & t_{k} \notin\left(T_{k-k}, T_{k}\right)
\end{array}\right.
$$

(здесь и далее $\left.T_{0}=0\right)$. Теперь вместо (20) можно ввести систему уравнений в частных производных с бесконечным набором координат $\mathbf{t}:=\left(t_{1}, t_{2}, \ldots\right)$ :

$$
\left(z-\xi_{k}\left(t_{k}\right)\right) \frac{\partial f}{\partial t_{k}}=P_{k}\left(z, t_{k}\right), \quad k=1,2, \ldots
$$


Предположим, что функция $f_{0}(z)$ разлагается в окрестности бесконечности следующим образом:

$$
f_{0}(z)=z+\sum_{n=0}^{\infty} \frac{A^{n}}{z^{n+1}},
$$

и пусть $f_{0}$ служит начальным условием для цепочки Лёвнера, управляемой уравнением (20), и начальным условием для первого уравнения системы (21). Следовательно, функция $f\left(z, t_{k}\right)$ выступает в качестве начальных данных для $(k+1)$-го уравнения системы (21). Ясно, что итоговая цепочка $f(z, t)=f(z, \mathbf{t}), \mathbf{t}=\left(t_{1}, t_{2}, \ldots\right)$, в противоположность цепочке, полученной из (20), является кусочно-дифференцируемой. Функции $f(z, t)$ нормированы так же, как и функци $(22)$ с $A^{n}=A^{n}(t)$.

Итак, существуют управляющие функции $\xi_{1}\left(t_{1}\right), \xi_{2}\left(t_{2}\right), \ldots$ такие, что функция $f(z, \mathbf{t}), \mathbf{t}=\left(t_{1}, t_{2}, \ldots\right)$, является решением бесконечной системы уравнений $(21)$ в частных производных.

Применим полученные в статье [7] результаты, чтобы построить для системы (21) редукции к уравнению от одной переменной для иерархии бКП.

Пусть $g(w, \mathbf{t}):=f^{-1}(w, \mathbf{t})$ - функция, обратная к $f(z, \mathbf{t})$. Тогда $g$ нормирована на бесконечности следующим образом:

$$
g(w, \mathbf{t})=w+\sum_{n=1}^{\infty} \frac{b_{n}(\mathbf{t})}{w^{n}} .
$$

Обозначим полиномы Файбера для $g(w, \mathbf{t})$ как $\Phi_{k}(w, \mathbf{t})=\left[g^{k}(w, \mathbf{t})\right]_{\geqslant 0}, k=1,2, \ldots$. Забудем на некоторое время про зависимость от $\mathbf{t}$ и напишем просто $g(w)$ и $\Phi_{k}(w)$. Первые полиномы Фабера имеют вид

$$
\Phi_{0}=1, \quad \Phi_{1}=w, \quad \Phi_{2}=w^{2}-2 b_{1}, \quad \Phi_{3}=w^{3}-3 b_{1} w-3 b_{2},
$$

а рекуррентная формула

$$
\Phi_{n+1}=w \Phi_{n}-\sum_{k=1}^{n-1} b_{n-k} \Phi_{k}-(n+1) b_{n}
$$

справедлива для всех $n=1,2, \ldots$. Полиномы Фабера связаны с коэффициентами Грунского, что влечет

$$
\ln \frac{g(w)-\xi}{w}=-\sum_{n=1}^{\infty} \frac{1}{n w^{n}} \Phi_{n}(\xi) .
$$

Производя замену переменной $\xi=e^{u}$ и дифференцируя обе части по $u$, получим

$$
\frac{1}{g(w)-\xi}=\sum_{n=1}^{\infty} \frac{1}{n w^{n}} \Phi_{n}^{\prime}(\xi) .
$$

Возвращаясь к уравнению (21), приходим к выводу, что функция $g=f^{-1}$ по первой переменной удовлетворяет системе уравнений

$$
\frac{\partial g}{\partial t_{k}}=\left\{\begin{array}{lll}
-\frac{\partial A^{0}}{\partial t_{k}} \sum_{n=1}^{\infty} \frac{\Phi_{n}^{\prime}\left(\xi_{k}, t_{k}\right)}{n w^{n}} & \text { при } \quad T_{k-1}<t_{k}<T_{k}, \\
0 & \text { при } \quad t_{k} \notin\left(T_{k-1}, T_{k}\right),
\end{array}\right.
$$


Это означает, что для всех $k=1,2, \ldots$

$$
n \frac{\partial b_{k}}{\partial t_{k}}=\left\{\begin{array}{lll}
-\frac{\partial A^{0}}{\partial t_{k}} \Phi_{k}^{\prime}\left(\xi_{k}, t_{k}\right) & \text { при } & T_{k-1}<t_{k}<T_{k}, \\
0 & \text { при } & t_{k} \notin\left(T_{k-1}, T_{k}\right) .
\end{array}\right.
$$

Существует очевидный способ написать зависимость от $\mathbf{t}$ через одну переменную $t=t_{1}$. Положим

$$
\tau(\mathbf{t})=\left\{\begin{array}{lll}
t_{1} & \text { при } & t_{1} \in\left(0, T_{1}\right), \\
k t_{k}\left(t_{1}\right) & \text { при } & t_{1} \in\left(T_{k-1}, T_{k}\right), \quad k=2,3, \ldots,
\end{array}\right.
$$

где

$$
\frac{d t_{k}}{d t_{1}}=\left\{\begin{array}{lll}
\Phi_{k}^{\prime}\left(\xi_{k}, t_{k}\right) & \text { при } & t_{1} \in\left(0, T_{1}\right), \\
0 & \text { при } & t_{1} \notin\left(T_{k-1}, T_{k}\right), \quad k=2,3, \ldots .
\end{array}\right.
$$

Проводя рассуждения в духе результатов Такебе, Тео и Забродина [7], заключаем следующее: непересекающиеся интервалы $\left(T_{k-1}, T_{k}\right)$ означают, что для функции $f(z, \tau(\mathbf{t}))$, заданной в качестве решения системы (21) с начальным условием $f_{0}$, мы имеем функцию Лакса $\mathcal{L}=f(z, \tau(\mathbf{t}))$, которая является решением иерархии бКП

$$
\frac{\partial \mathcal{L}}{\partial t_{k}}=\left\{\mathcal{L}_{k}, \mathcal{L}\right\}, \quad T_{k-1}<t_{k}<T_{k},
$$

где $\mathcal{L}_{k}=k^{-1}\left[\mathcal{L}^{k}\right]_{\geqslant 0}$, а скобка Пуассона задана в виде

$$
\{F, G\}=\frac{\partial F}{\partial w} \frac{\partial G}{\partial x}-\frac{\partial F}{\partial x} \frac{\partial G}{\partial w}, \quad T_{k-1}<x:=t_{1}<T_{k} .
$$

Уравнение Бенни снова можно восстановить как второе уравнение иерархии бКП следующим образом. Положим $s=t_{2}$. Тогда

$$
\frac{\partial \mathcal{L}}{\partial s}=\left\{z^{2}+2 A^{0}, \mathcal{L}\right\}, \quad T_{1}<s, x<T_{2},
$$

где

$$
\mathcal{L}=z+\sum_{n=0}^{\infty} \frac{A^{n}}{z^{n+1}} .
$$

Приравнивая коэффициенты перед степенями $z$, приходим к уравнениям (12). Высшие уравнения иерархии дают интересные уравнения в частных производных благодаря условиям на коммутирующие потоки (условиям совместности) (3). Например, при $n=2$ и $m=3$ мы приходим к уравнению бКП (4) (уравнению Хохлова-Заболотской [3]) для $A^{0}$.

\section{5. КИНЕТИЧЕСКОЕ УРАВНЕНИЕ ВЛАСОВА}

Вернемся к условиям совместности (6) (системе Гиббонса-Царева), где $u(\mathbf{r})$ плотность закона сохранения, а $\mu_{k}(\mathbf{r})$ - характеристические скорости для $N$-компонентной системы гидродинамического типа

$$
r_{s}^{i}+\mu_{i}(\mathbf{r}) r_{x}^{i}=0, \quad i=1, \ldots, N,
$$


написанной в инвариантах Римана. Система (23) интегрируема с помощью обобщенного метода годографа [21], [22] и имеет бесконечное число законов сохранения и коммутирующих потоков

$$
r_{y}^{i}+\lambda_{i}(\mathbf{r}) r_{x}^{i}=0
$$

где $\lambda_{i}-$ функции, которые зависят только от двух переменных $\mu_{i}, u$, а именно $\lambda_{i}=F\left(\mu_{i}, u\right)$. Воспользуемся системой Царева

$$
\frac{\partial_{i} \lambda_{k}}{\lambda_{i}-\lambda_{k}}=\frac{\partial_{i} \mu_{k}}{\mu_{i}-\mu_{k}}, \quad i \neq k,
$$

которая является прямым следствием равенства $\left(r_{t}^{i}\right)_{y}=\left(r_{y}^{i}\right)_{t}$. Подстановка представления $\lambda_{i}=F\left(\mu_{i}, u\right)$ в (25) дает

$$
\left(\mu_{k}-\mu_{i}\right) \frac{\partial F\left(\mu_{i}, u\right)}{\partial u}=\frac{F\left(\mu_{k}, u\right)-F\left(\mu_{i}, u\right)}{\mu_{k}-\mu_{i}}-\frac{\partial F\left(\mu_{i}, u\right)}{\partial \mu_{i}}, \quad i \neq k .
$$

Суммируя равенство (26) с равенством, полученным из (26) путем перестановки индексов, выводим уравнение

$$
\left(\mu_{k}-\mu_{i}\right)\left(\frac{\partial F\left(\mu_{i}, u\right)}{\partial u}+\frac{\partial F\left(\mu_{k}, u\right)}{\partial u}\right)=\frac{\partial F\left(\mu_{k}, u\right)}{\partial \mu_{k}}-\frac{\partial F\left(\mu_{i}, u\right)}{\partial \mu_{i}} .
$$

Эта формула в пределе $\mu_{k} \rightarrow \mu_{i}$ принимает вид

$$
2 \frac{\partial F\left(\mu_{i}, u\right)}{\partial u}=\frac{\partial^{2} F\left(\mu_{i}, u\right)}{\partial \mu_{i}^{2}} .
$$

Тогда уравнение (27) переходит в

$$
\left(\mu_{k}-\mu_{i}\right)\left(\frac{\partial^{2} F\left(\mu_{i}, u\right)}{\partial \mu_{i}^{2}}+\frac{\partial^{2} F\left(\mu_{k}, u\right)}{\partial \mu_{k}^{2}}\right)=2\left(\frac{\partial F\left(\mu_{k}, u\right)}{\partial \mu_{k}}-\frac{\partial F\left(\mu_{i}, u\right)}{\partial \mu_{i}}\right) .
$$

Взяв производную этого выражения по $\mu_{k}$, получим

$$
\frac{\partial^{3} F\left(\mu_{k}, u\right)}{\partial \mu_{k}^{3}}=\frac{1}{\mu_{k}-\mu_{i}}\left(\frac{\partial^{2} F\left(\mu_{k}, u\right)}{\partial \mu_{k}^{2}}-\frac{\partial^{2} F\left(\mu_{i}, u\right)}{\partial \mu_{i}^{2}}\right), \quad i \neq k .
$$

Переставляя индексы, приходим к выводу, что

$$
\frac{\partial^{3} F\left(\mu_{k}, u\right)}{\partial \mu_{k}^{3}}=a^{\prime}(u)
$$

для любого индекса $k$. Таким образом,

$$
F\left(\mu_{k}, u\right)=\frac{1}{6} a(u) \mu_{k}^{3}+b(u) \mu_{k}^{2}+c(u) \mu_{k}+d(u),
$$

где функции $a(u), b(u), c(u)$ и $d(u)$ все еще не определены. Однако подстановка (29) в равенство (28) приводит к

$$
a(u)=\text { const }, \quad b(u)=\text { const }, \quad 2 c^{\prime}(u)=a, \quad d^{\prime}(u)=b .
$$


В результате выражение (29) принимает вид

$$
F\left(\mu_{k}, u\right)=\frac{a}{6} \mu_{k}^{3}+\frac{a}{2} u \mu_{k}+b\left(\mu_{k}^{2}+u\right) .
$$

Наконец, подставив эту формулу в (26), получим $a=0$. Таким образом, мы нашли так называемое "дисперсионное соотношение"

$$
\lambda_{i}=\mu_{i}^{2}+u
$$

Согласно [22] напишем систему Лёвнера

$$
\partial_{i} z=\frac{\partial_{i} u}{\mu_{i}-z}
$$

Тогда с учетом соотношений $(23),(24),(31),(32)$ получаем

$$
\begin{aligned}
z_{x} & =\sum \partial_{i} z \cdot r_{x}^{i}=\sum \frac{\partial_{i} u}{\mu_{i}-z} r_{x}^{i} \\
-z_{s} & =-\sum \partial_{i} z \cdot r_{s}^{i}=\sum \frac{\partial_{i} u}{\mu_{i}-z} \mu_{i} r_{x}^{i}=\sum \partial_{i} u \cdot r_{x}^{i}+z \sum \frac{\partial_{i} u}{\mu_{i}-z} r_{x}^{i}=u_{x}+z z_{x} \\
-z_{y} & =-\sum \partial_{i} z \cdot r_{y}^{i}=\sum \frac{\partial_{i} u}{\mu_{i}-z} \lambda_{i} r_{x}^{i}=\sum \frac{\partial_{i} u}{\mu_{i}-z}\left(\mu_{i}^{2}+u\right) r_{x}^{i}= \\
& =\sum \partial_{i} u \cdot\left(\mu_{i}-z\right) r_{x}^{i}+2 z \sum \partial_{i} u \cdot r_{x}^{i}+\left(z^{2}+u\right) \sum \frac{\partial_{i} u}{\mu_{i}-z} r_{x}^{i}= \\
& =\left(v_{x}-z u_{x}\right)+2 z u_{x}+\left(z^{2}+u\right) z_{x}=v_{x}+z u_{x}+u z_{x}+z^{2} z_{x}
\end{aligned}
$$

где введена новая функция $v(\mathbf{r})$ такая, что $\partial_{i} v=\mu_{i} \partial_{i} u$. В самом деле, потенциал $v$ существует, поскольку условие совместности $\partial_{i}\left(\partial_{k} v\right)=\partial_{k}\left(\partial_{i} v\right)$ приводит к указанному тождеству в соответствии с системой Гиббонса-Царева (6).

Таким образом, мы восстановили два уравнения

$$
z_{s}+\left(\frac{z^{2}}{2}+u\right)_{x}=0, \quad z_{y}+\left(\frac{z^{3}}{3}+u z+v\right)_{x}=0 .
$$

Условие их совместности $\left(z_{s}\right)_{y}=\left(z_{y}\right)_{s}$ приводит к уравнениям

$$
v_{x}+u_{s}=0, \quad v_{s}+u_{y}+u u_{x}=0,
$$

которые эквивалентны уравнению (4).

Теперь введем так называемое вершинное уравнение

$$
\partial_{\tau(\zeta)} z(\lambda)=\partial_{x} \ln (z(\lambda)-z(\zeta))
$$

где $z(\lambda)$ - сокращенное обозначение для $z\left(\lambda ; t_{0}, t_{1}, t_{2}, \ldots\right)$. Здесь мы используем бесконечно много временны́х переменных $t_{k}$, которые обсуждаются ниже. Рассмотрим формальное разложение (15) при $\zeta \rightarrow \infty$ и разложение

$$
\partial_{\tau(\zeta)}=-\frac{1}{\zeta} \partial_{t_{0}}-\frac{1}{\zeta^{2}} \partial_{t_{1}}-\frac{1}{\zeta^{3}} \partial_{t_{2}}-\cdots
$$


Тогда можно получить бесконечный набор уравнений

$$
\partial_{t_{n}} z(\lambda)=\partial_{x} \frac{\Phi_{n+1}(z(\lambda))}{n+1}, \quad n=0,1,2 \ldots,
$$

где $\Phi_{n}$ означают полиномы Фабера (см. раздел 4). Для первых полиномов имеем

$$
\begin{gathered}
\partial_{t_{0}} z(\lambda)=z_{x}(\lambda), \quad \partial_{t_{1}} z(\lambda)=\left(\frac{z^{2}(\lambda)}{2}+H^{0}\right)_{x}, \\
\partial_{t_{2}} z(\lambda)=\left(\frac{z^{3}(\lambda)}{3}+H^{0} z(\lambda)+H^{1}\right)_{x},
\end{gathered}
$$

где мы провели отождествления $x=t_{0}, s=-t_{1}, y=-t_{2}$, а также $u=H^{0}, v=H^{1}$ (см. уравнения (33)).

Подставляя аналогичное формальное разложение (при $\lambda \rightarrow \infty)$

$$
z(\lambda)=\lambda-\frac{H^{0}}{\lambda}-\frac{H^{1}}{\lambda^{2}}-\frac{H^{2}}{\lambda^{3}}-\cdots
$$

в эти производящие функции законов сохранения, получим бесконечное число локальных законов сохранения. Например,

$$
\left(H^{k}\right)_{s}+\left(H^{k+1}-\frac{1}{2} \sum_{m=0}^{k-1} H^{m} H^{k-1-m}\right)_{x}=0, \quad k=0,1,2, \ldots
$$

Это есть не что иное, как гидродинамическая цепочка Бенни (2), представленная в консервативной форме, когда все плотности законов сохранения $H^{k}$ полиномиальны по моментам $A^{m}$, как в разделе 2 .

Альтернативные разложения (при $\zeta \rightarrow 0$ )

$$
z(\zeta)=H^{-1}+\zeta H^{-2}+\zeta^{2} H^{-3}+\cdots, \quad \partial_{\tau(\zeta)}=\partial_{t_{-1}}+\zeta \partial_{t_{-2}}+\zeta^{2} \partial_{t_{-3}}+\cdots
$$

приводят к другим производящим функциям законов сохранения, например

$$
\partial_{t_{-1}} z(\lambda)=\partial_{x} \ln \left(z(\lambda)-H^{-1}\right), \quad \partial_{t_{-2}} z(\lambda)=-\partial_{x} \frac{H^{-2}}{z(\lambda)-H^{-1}} .
$$

Подстановка разложения (37) и разложения (при $\lambda \rightarrow 0$ )

$$
z(\lambda)=H^{-1}+\lambda H^{-2}+\lambda^{2} H^{-3}+\cdots
$$

приводит к бесконечному числу дополнительных законов сохранения (ср. с (38)). Если, например, подставить приведенное выше разложение в (33), то получим два дополнительных закона сохранения

$$
\begin{aligned}
& \partial_{s} H^{-1}=-\left(\frac{1}{2}\left(H^{-1}\right)^{2}+H^{0}\right)_{x}=-\partial_{x} \frac{\Phi_{2}\left(H^{-1}\right)}{2} \\
& \partial_{y} H^{-1}=-\left(\frac{1}{3}\left(H^{-1}\right)^{3}+H^{0} H^{-1}+H^{1}\right)_{x}=-\partial_{x} \frac{\Phi_{3}\left(H^{-1}\right)}{3} .
\end{aligned}
$$


Бесконечно большое число законов сохранения (ср. с (38))

$$
\begin{gathered}
\left(H^{-1}\right)_{s}+\left(H^{0}+\frac{1}{2}\left(H^{-1}\right)^{2}\right)_{x}=0, \\
\left(H^{k}\right)_{s}+\left(H^{k+1}-\frac{1}{2} \sum_{m=0}^{k-1} H^{m} H^{k-1-m}\right)_{x}=0, \quad k=0,1,2, \ldots,
\end{gathered}
$$

можно также представить в виде модифицированной гидродинамической цепочки Бенни (подробности см. в работах [24])

$$
B_{s}^{k}+B_{x}^{k+1}+\frac{1}{2} B^{0} B_{x}^{k}+\frac{k+1}{2} B^{k} B_{x}^{0}+k B^{k-1}\left(\frac{1}{2} B^{1}-\frac{1}{8}\left(B^{0}\right)^{2}\right)_{x}=0,
$$

где

$$
H^{-1}=B^{0}, \quad H^{0}=B^{1}, \quad H^{1}=B^{2}+B^{0} B^{1}-\frac{1}{12}\left(B^{0}\right)^{3}, \ldots
$$

Такая модифицированная гидродинамическая цепочка Бенни связана с модифицированным уравнением бКП (ср. с (34))

$$
H_{s}^{-1}+\left(H^{0}+\frac{1}{2}\left(H^{-1}\right)^{2}\right)_{x}=0, \quad H_{s}^{0}=H_{y}^{-1}+\left(H^{0} H^{-1}+\frac{1}{3}\left(H^{-1}\right)^{3}\right)_{x},
$$

которое можно получить из условия совместности $\left(\tilde{z}_{s}\right)_{y}=\left(\tilde{z}_{y}\right)_{s}$, где $\tilde{z}$ удовлетворяет уравнениям

$$
\tilde{z}_{s}+\left(\frac{\tilde{z}^{2}}{2}+H^{-1} \tilde{z}\right)_{x}=0, \quad \tilde{z}_{y}+\left(\frac{\tilde{z}^{3}}{3}+H^{-1} \tilde{z}^{2}+\left(H^{0}+\left(H^{-1}\right)^{2}\right) \tilde{z}\right)_{x}=0 .
$$

Можно вывести модифицированные уравнения Лёвнера

$$
\partial_{i} \tilde{z}=\tilde{z} \frac{\partial_{i} H^{-1}}{\mu_{i}-\tilde{z}-H^{-1}},
$$

которые эквивалентны исходным уравнениям Лёвнера (32) благодаря подстановкам $z=\tilde{z}+H^{-1}$ и $\left(\mu_{i}-H^{-1}\right) \partial_{i} H^{-1}=\partial_{i} H^{0}$.

Обе гидродинамические цепочки имеют одну и ту же локальную гамильтонову структуру

$$
\begin{aligned}
& A_{s}^{k}=-\frac{1}{2}\left[(k+m) A^{k+m-1} \partial_{x}+m A_{x}^{k+m-1}\right] \frac{\partial H^{2}}{\partial A^{m}}, \\
& B_{s}^{k}=-\frac{1}{2}\left[(k+m) B^{k+m-1} \partial_{x}+m B_{x}^{k+m-1}\right] \frac{\partial H^{1}}{\partial B^{m}},
\end{aligned}
$$

где плотности гамильтонианов суть

$$
H^{2}=A^{2}+\left(A^{0}\right)^{2}, \quad H^{1}=A^{1}=B^{2}+B^{0} B^{1}-\frac{1}{12}\left(B^{0}\right)^{3} .
$$

Поскольку все моменты $A^{k}$ можно выразить через моменты $B^{0}, B^{1}, \ldots, B^{k}, B^{k+1}$, скобки Купершмидта-Манина

$$
\left\{B^{k}, B^{m}\right\}=\left((k+m) B^{k+m-1} \partial_{x}+m B_{x}^{k+m-1}\right) \delta\left(x-x^{\prime}\right)
$$


можно пересчитать через моменты $A^{s}$. Это означает, что гидродинамическая цепочка Бенни имеет по крайней мере две локальные гамильтоновы структуры (подробности см. в работах [24]).

Как и в предыдущем частном случае, будем искать $N$-компонентные коммутирующие гидродинамические редукции

$$
r_{\tau(\zeta)}^{i}=w^{i}(\mathbf{r}, \zeta) r_{x}^{i}
$$

Тогда уравнение (35) сводится к

$$
\partial_{i} z(\lambda)=\frac{\partial_{i} z(\zeta)}{1-(z(\lambda)-z(\zeta)) w^{i}(\mathbf{r}, \zeta)} .
$$

Принимая во внимание уравнение (32), можно получить, что

$$
w^{i}(\mathbf{r}, \zeta)=\frac{1}{\mu_{i}-z(\zeta)}
$$

Используя разложения (36) и (39), мы можем разложить производящую функцию (по параметру $\zeta$ в окрестности бесконечности и около нуля соответственно) бесконечного числа высших коммутирующих потоков

$$
r_{\tau(\zeta)}^{i}=\frac{1}{\mu_{i}-z(\zeta)} r_{x}^{i}
$$

Благодарности. М.В. Павлов получил финансовую поддержку Программы Президиума РАН "Фундаментальные проблемы нелинейной динамики" и РФФИ (грант № 14-01-00389). А. Ю. Васильев финансово поддержан Norwegian Research Council (гранты № 204726/V30, 213440/BG), а также EU FP7 IRSES Programm STREVCOMS (грант № PIRSES-GA-2013-612669).А.М. Захаров и Д. В. Прохоров получили финансовую поддержку Министерства науки и образования РФ (грант $1.1520 .2014 \mathrm{k})$.

\section{Список литературы}

[1] Y. Kodama, J. Gibbons, Phys. Lett. A, 135:3 (1989), 167-170.

[2] D. J. Benney, Stud. Appl. Math., 52:1 (1973), 45-50.

[3] Е. А. Заболотская, Р. В. Хохлов, Акустический журн., XV:1 (1969), 40-47.

[4] В. Е. Захаров, Функи. анализ и его прил., 14:2 (1980), 15-24.

[5] J. Gibbons, S. Tsarev, Phys. Lett. A, 258:4-6 (1999), 263-271.

[6] M. Mañas, L. Martínez Alonso, E. Medina, J. Phys. A, 35:2 (2002), 401-417.

[7] T. Takebe, L.-P. Teo, A. Zabrodin, J. Phys. A, 39:37 (2006), 11479-11501.

[8] B. Gustafsson, A. Vasil'ev, Conformal and Potential Analysis in Hele-Shaw Cells, Birkhäuser, Basel, 2006.

[9] S. Richardson, J. Fluid. Mech., 56:4 (1972), 609-618.

[10] M. Mineev-Weinstein, P. B. Wiegmann, A. Zabrodin, Phys. Rev. Lett., 84:22 (2000), $5106-5109$.

[11] I. Markina, A. Vasil'ev, "Virasoro algebra and dynamics in the space of univalent functions" (Sevilla, Spain, February 5-9, 2008), Contemporary Mathematics, 525 (2010), 85-116.

[12] M. V. Pavlov, Int. Math. Res. Not., 2006:20 (2006), 46987, 43 pp. 
[13] A. A. Vlasov, Many-Particle Theory and Its Application to Plasma, Gordon and Breach, New York, 1961.

[14] M. V. Pavlov, S.P. Tsarev, Classical mechanical systems with one-and-a-half degrees of freedom and Vlasov kinetic equation, arXiv: 1306.3737; Amer. Math. Soc. Transl. Ser. 2, 2014 (to appear).

[15] Б. А. Купершмидт, Ю. И. Манин, Функи. анализ и его прил., 11:3 (1977), 31-42.

[16] M. V. Pavlov, Commun. Math. Phys., 272:2 (2007), 469-505.

[17] J. Gibbons, Phys. Lett. A, 90:1-2 (1982), 7-8.

[18] O. Tammi, Ann. Acad. Sci. Fenn. Ser. A. I. Math., 13:1 (1988), 125-136.

[19] М. В. Павлов, С. П. Царев, УМH, 46:4(280) (1991), 169-170.

[20] Б. А. Купершмидт, Ю. И. Манин, Функи. анализ и его прил., 12:1 (1978), 25-37.

[21] С. П. Царев, Докл. АН СССР, 282:3 (1985), 534-537.

[22] С. П. Царев, Изв. АН СССР. Сер. матем., 54:5 (1990), 1048-1068.

[23] Е. В. Ферапонтов, частное сообщение.

[24] B. A. Kupershmidt, Proc. Roy. Irish Acad. Sect. A, 83:1 (1983), 45-74; "Normal and universal forms in integrable hydrodynamical systems", Proceedings of Berkeley-Ames Conference on Nonlinear Problems in Control and Fluid Dynamics (Berkeley, CA, 1983), Lie Groups: History, Frontiers and Applications, Series B: Systems Information and Control, eds. L. R. Hunt, C. F. Martin, Math. Sci. Press, Brookline, MA, 1984, 357-378.

Поступила в редакцию 4.06.2014 